\title{
The Egyptian mongoose, Herpestes ichneumon, is a possible reservoir host of visceral leishmaniasis in eastern Sudan
}

\author{
D. A. ELNAIEM ${ }^{1,2}{ }^{2} \dagger$, M. M. HASSAN ${ }^{1}$, R. MAINGON ${ }^{2}$, G. H. NURELDIN ${ }^{1}$, \\ A. M. MEKAWI, M. M I LES ${ }^{3}$ and R. D. WARD ${ }^{2}$ \\ ${ }^{1}$ Department of Zoology, Faculty of Science, University of Khartoum, Sudan \\ ${ }^{2}$ School of Life Sciences, Keele University, Staffordshire ST5 5BG, UK \\ ${ }^{3}$ Pathogen Molecular Biology and Biochemistry Unit, Department of Infectious and Tropical Diseases, \\ London School of Hygiene and Tropical Medicine, Keppel Street, London WC1E 7HT, UK
}

(Received 8 Fune 2000; revised 23 October 2000 ; accepted 15 November 2000)

SUMMARY

Investigations were made on possible reservoir hosts of Leishmania donovani in 2 zoonotic foci of visceral leishmaniasis (VL) in Dinder National Park (DNP) and the peri-domestic habitats of adjacent villages of eastern Sudan. Animals were captured, in November 1997-1998 and April-May 1999 and examined for L. donovani infection using light microscopy and 2 sensitive Polymerase Chain Reaction (PCR) systems. Microscopy and PCR investigations were also used to determine the infection rates of $L$. donovani in Phlebotomus orientalis captured from the uninhabited site of DNP. Infections of L. donovani were detected in 2 out of 14 Egyptian mongooses (Herpestes ichneumon), 1 out of 168 Arviconthus niloticus and 1 out of 8 Mastomys natalensis. Samples from 68 other animals captured from the study area were all negative for the infection. Active zoonotic transmission of L. donovani at the time of animal sampling in the uninhabited site of DNP was demonstrated by finding the parasite in $3.4 \%$ (7 out of 184) and 3.2\% (5 out of 157) of flies collected in March 1998 and May 1999, respectively. We suggest that the Egyptian mongoose is a possible reservoir host of L. donovani. The importance of other animals in maintaining the infection is also discussed.

Key words: Leishmania donovani, sandflies, reservoir, mongoose, Sudan.

\section{INTRODUCTION}

Members of the Leishmania donovani complex are the causative agents of visceral leishmaniasis (VL), a lethal disease endemic in many tropical countries (Desjeux, 1996). The parasite has a heteroxenous life-cycle involving mammalian hosts and phlebotomine sandfly vectors (Killick-Kendrick, 1992). The epidemiology of $\mathrm{VL}$ varies from one region to another, depending on the ecology of endemic areas, demographic factors, parasite strains, vector species and the presence or absence of reservoir hosts (reviewed by Peters \& Killick-Kendrick, 1987). In the New World, VL occurs as a zoonosis, involving the domestic dog (Canis familiaris) and foxes (Dusicyon vetulus and Cerdocyon thous) (Lainson, Shaw \& Lins, 1969; Lainson, 1983; Courtney et al. 1996). Similarly, in the Mediterranean region, VL transmission involves canine reservoir hosts, mainly the dog and several fox species, including Vulpes vulpes, $V$. corsac and $V$. zerda (reviewed by Ashford

* Corresponding author: School of Life Sciences, Keele University, Staffordshire ST5 5BG, UK. Tel: + 441782 58 3417. Fax: +44 1782583516.

E-mail: dialnaiem@hotmail.com

$\uparrow$ Permanent address: Department of Zoology, Faculty of Science, University of Khartoum, Khartoum, P O Box 321, Sudan.
\& Bettini, 1987 and Ashford, 1996). In contrast, the transmission of $\mathrm{VL}$ in the Indian subcontinent is exclusively anthroponotic. Although anthroponotic transmission of VL may also exist in East Africa, especially during epidemic situations, it is thought that these epidemics must arise from residual zoonotic foci with unknown reservoir hosts (Ashford, 1996).

Visceral leishmaniasis, caused by 3 zymodemes of L. donovani (Ashford et al. 1992; Oskam et al. 1998), and transmitted by $P$. orientalis (Hoogstraal \& Heyneman, 1969; Schorscher \& Goris, 1993; Elnaiem, Hassan \& Ward, 1997; Elnaiem et al. 1998; Elnaeim \& Osman, 1998), is endemic over a large area in eastern and southern Sudan with severe epidemics, which result in high mortality and morbidity figures. In a recent outbreak in southern Sudan, VL claimed the lives of 100000 people out of a population of 300000 living in a relatively small area in the Western Upper Nile Province, southern Sudan (Seaman, Mercer \& Sondorp, 1996). Similarly, over 11000 cases of VL have occurred over the past 3 years among approximately 200000 people living in Gedaref State in eastern Sudan (Reports of Medicins Sans Frontier-Holland, Khartoum Office). From repeated outbreaks of VL in people camping in uninhabited woodlands of eastern and southern Sudan, it has long been suggested that the parasite is 
maintained in a reservoir host (Kirk, 1939). Although few silvatic mammals were found infected with equivocally identified Leishmania parasites (Hoogstraal \& Heyneman, 1969; Sixl et al. 1987), no animal species has been incriminated as a VL reservoir host (Ashford, 1996). Recent studies also detected L. donovani infection in dogs (Dereure et al. 2000). However, infections in dogs, which are only limited to the villages, cannot explain the flare up of the disease in people camping in uninhabited woodland (Kirk, 1939).

We have recently provided conclusive evidence for the existence of a silvatic reservoir host of $\mathrm{VL}$ in eastern Sudan by finding that up to $6.9 \%$ of the population of $P$. orientalis of an uninhabited site in Dinder National Park (DNP) were infected by $L$. donovani (Elnaiem et al. 1998). Since neither people nor domestic animals live in this site, it was clear that the parasite was circulating between silvatic animals, which have close association with the vector. In the present study, we re-visited the same site in DNP and other adjacent areas, in eastern Sudan, to study the small mammal fauna of the area and investigate their possible role as reservoir hosts of $L$. donovani.

\section{MATERIALS AND METHODS}

\section{Study area}

The field study was carried out in 2 main localities in eastern Sudan; one site in a thicket of Acacia seyal in $\mathrm{DNP}\left(35^{\circ} 2^{\prime} \mathrm{E}, 12^{\circ} 36^{\prime} \mathrm{N}\right)$ and the other site in the peri-domestic habitat of Ein-Elgamel and Umsalala villages (approx $35^{\circ} 11^{\prime} \mathrm{E}, 12^{\circ} 51^{\prime} \mathrm{N}$ ), close to the eastern bank of the River Rahad. The location and ecology of the study area were described in a previous publication (Elnaiem et al. 1997). The two sites fall within the region of maximum risk of VL described by Thomson et al. (1999). The area of the villages was reported to have an annual incidence of $38 \mathrm{VL}$ cases per 1000 (Zijlstra et al. 1994). Similarly, many visitors contract the disease, when staying for a few days in DNP.

\section{Animal trapping and sampling methods}

Small mammals from the area were sampled using 3 trapping methods. Rodents of the family Muridae were captured by live-multi-traps, using baits consisting of vegetables and peanut butter. In addition to this method, large numbers of the Nile rat (Arvicanthis niloticus) were also captured by stringtraps designed by local hunters and placed on rodent paths within the grass. Squirrels were captured, by chasing them from their burrows. Carnivores were captured using locally made spring-traps, which were baited with fish or meat. Although observations on the presence and distribution of small mammals in the area included all possible species, the animals captured and screened for the presence of Leishmania infections were mainly rodents, carnivores and insectivores. Endangered or rare species, such as the civet or the serval were identified and released. All animal samplings were carried out in 2 consecutive dry seasons, during November 1997-March 1998 and then April-May 1999.

\section{Dissection of animals and preservation of tissue samples for microscopy and polymerase chain reaction $(P C R)$ detection of Leishmania parasites}

Captured animals were killed and dissected to obtain samples from spleen, liver and skin for screening for presence of Leishmania. In the first season (19971998), impression smears made from autopsy samples of the spleen and liver tissues of dissected animals were fixed in methanol and preserved for later staining and microscopy. In each of the two seasons, duplicate samples from the spleen, liver and skin were preserved in $95 \%$ isopropanol for later PCR screening for presence of Leishmania DNA. Skins and skulls from representative samples of all captured animals were preserved and later identified to species, at the British Museum of Natural History.

\section{Microscopical examination and PCR analysis of tissues samples}

The methanol-fixed impression smears were stained by Giemsa's stain and examined under $\times 100$ oilimmersion lens for presence of Leishmania amastigotes. Three investigators examined 20 microscopic fields of each spleen or liver smear.

To prepare DNA extracts for PCR screening, small portions of the isopropanol preserved specimens (approximately $5 \mathrm{~mm}$ in diameter) of spleen and skin tissues were air dried, chopped into small fragments, lysed in buffer containing proteinase-K and subjected to standard phenol/ chloroform purification and ethanol/salt precipitation procedure. Precipitated DNA was washed $\times 3$ in $70 \%$ ethanol, air dried and then dissolved in $100 \mu \mathrm{l}$ of PCR water, overnight, and stored at $4{ }^{\circ} \mathrm{C}$. To avoid contamination between specimens, each sample was processed separately using a new set of blades and plastic-ware.

Two PCR systems were used to examine the spleen and skin samples of animals for the presence of DNA of L. donovani. Initially, the nested PCRsystem designed by Noyes et al. (1998) was used to detect the presence of Leishmania kinetoplast DNA in all purified samples. The PCR conditions and primers used were the same as those described by Noyes et al. (1998). DNA extracts of $10^{2}$ promastigotes of L. donovani (MON18) parasites and spleen of a laboratory infected hamster were 
used as 2 positive controls. Negative controls were DNA of $10^{2}$ L. major promastigotes and 2 distilled water samples. Each DNA sample was tested twice at full concentration and once as a dilution of $1: 5$ in distilled water (total of 3 tests). In the case of positive samples, the nested-PCR was repeated twice on a fresh DNA extract from the original material.

Confirmed nested PCR-positive samples were retested by the L. donovani specific primers AJS31 (5'GGG GT'T GGT GTA AAA TAG GGC CCG 3') and DBY (5' CCA G'T'T TCC CGC CCC GGA G$\left.3^{\prime}\right)$, which were originally developed by Smyth et al. (1992), and then modified by the addition of the last 3 extra bases at the $3^{\prime}$ terminal end of each primer. The PCR conditions were similar to those described by Smyth et al. (1992).

The total number of animals examined by microscopy and PCR were 72 Arvicanthis niloticus, 19 Acomys albigena, 6 Mastomys natelensis, 3 Euxerus erythropus, 7 Atelerix albiventrix, 2 Crocidura oliveiri, 11 Herpestes ichneumon, 3 Ichneumonia albicauda, 2 Genetta genetta and 1 Ictonyx striatus. The total numbers of animals examined by PCR only were $96 \mathrm{~A}$. niloticus, $2 \mathrm{M}$. natalensis, $14 \mathrm{E}$. erythropis, 7 At. albiventrix, $3 \mathrm{H}$. ichneumon, $4 \mathrm{G}$. genetta and 6 I. striatus. The samples of A. niloticus, $C$. oliveiri and $G$. genetta were equally divided between the village and the DNP sites. In contrast all Ac. albigena specimens were obtained from the DNP site and all samples of E. erythropus, At. albiventrix, and $I$. striatus were collected from the village peri-domestic habitat.

Determination of the prevalence of $\mathrm{L}$. donovani infection in the vector population of the DNP site during the same season of animal sampling

Intensity of transmission of L. donovani in DNP during the 2 study seasons was investigated by determining the infection rates of the parasite in $P$. orientalis. Using our previously described methods (Elnaiem \& Osman, 1998), P. orientalis females captured by light traps set out in March 1998 and May 1999 were dissected and examined by microscopy and PCR for the presence of $L$. donovani infections.

\section{RESULTS}

\section{Small mammal fauna of the area}

During the course of the field study, a total of 405 small mammals, comprising 4 orders and 12 families, were captured and identified. In addition to the list of animals investigated for $L$. donovani infection, several other species of small mammals were recorded from the area. These included the Aardvark (Orycteropodidae: Orycteropus orycteropus), the honey badger (Mustillidae: Mellivora capenensis), the civet (Viverridae: Civeticttictis civetta), the caracal (Felidae: Felis caracal), the serval cat (Felidae: Felis serval), unidentified porcupine (Hystricidae, most probably Hystrix cristata), jackal (Canidae: most probably Canis adustus) and grivet monkey (Cercopithecidae: Cercopithecus aethiops) and unidentified baboons (Cercopithecidae). It was noticeable that throughout the study area, the most abundant small mammal species were the Nile Rat, A. niloticus, and the Egyptian mongoose, H. ichneumon. However, in certain pockets around the villages, abundant ground squirrels (Euxerus erythropus) and the hedgehogs (Atelerix albiventrix) were encountered. Trapping of mongooses, jackals and other carnivores was limited by several logistic problems; thus our collection does not reflect actual abundance of these animals, especially the mongoose which was a common sight in the area.

Microscopical detection of Leishmania infection in animals

Of all animals examined by microscopy, positive Leishmania infections were observed in 1 out of the 11 Egyptian mongooses collected in 1998. In this animal, at least 1 amastigote was noticeable in each microscopic field of the spleen smear. Some amastigotes were also observed in the liver smear of the same animal. Impression smears made from other small mammals were all negative for amastigote infection.

\section{PCR detection of $\mathrm{L}$. donovani $D N A$ in animal tissues}

Using the nested PCR technique, DNA of Leishmania parasites was consistently detectable in the spleen sample of the microscopically positive mongoose, and 1 of the 3 Egyptian mongooses trapped in the village peri-domestic habitat in 1999. Positive Leishmania DNA amplification was obtained from spleens of 2 other mongooses captured in 1998 in Dinder Park, but no PCR products were obtained when these samples were re-examined in 2 extra reactions on fresh extracts.

DNA of Leishmania parasites was also detectable in 1 out of the $6 M$. natalensis captured in DNP during 1998, and 1 out of 36 A. niloticus captured in 1998 in DNP. All skin samples and all other spleen samples gave no Leishmania specific PCR products.

In all positive samples, the mean ( \pm s.D.) size of the band obtained for $L$. donovani was $752 \pm 25 \mathrm{bp}$, which was clearly higher than a main band of a $643 \pm 19$ bp obtained for L. major (Fig. 1).

Results of PCR amplification of Leishmania kinetoplast DNA from the animal spleen samples, using the L. donovani specific primers, AJS31/DB are 


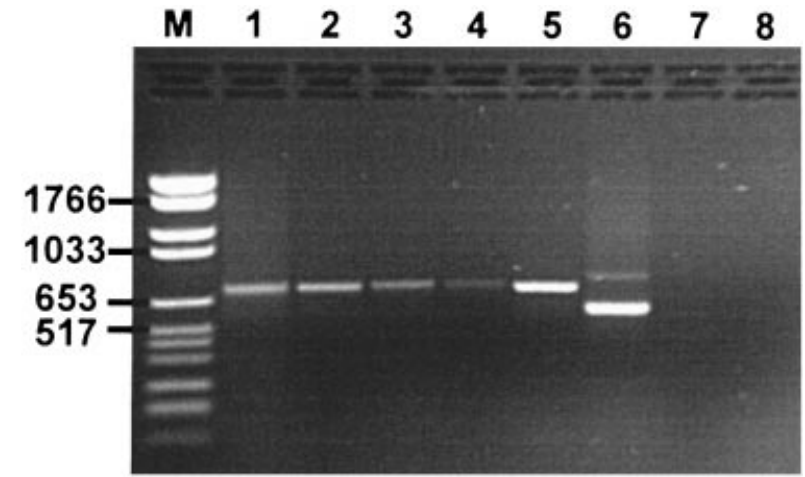

Fig. 1. Nested PCR amplification of kinetoplast DNA of Leishmania parasites in spleen samples of animals captured in a thicket of Acacia seyal in Dinder National Park and the peri-domestic habitats of Umsalala and Ein-Elgamel villages, eastern Sudan; M, DNA molecular weight marker; lane 1 , samples from mongoose captured in 1998 in DNP; lane 2, sample from mongoose captured in 1999 in peri-domestic habitat of Umsalala village; lane 3, sample from Mastomys natalensis captured in 1998 in DNP; lane 4, sample from Arvicanthis niloticus captured in 1999 in DNP; lane 5, DNA of $10^{2}$ promastigotes of $L$. donovani (MON18); lane 6, DNA of L. major; Lanes 7 and 8, PCR products from 2 samples of $\mathrm{H}_{2} \mathrm{O}$ (negative control).

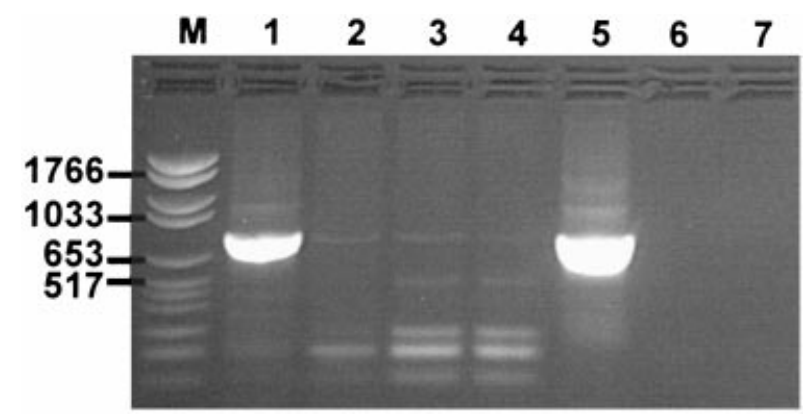

Fig. 2. PCR amplification of Leishmania donovanispecific kinetoplast minicircle DNA from spleen samples of animals captured in a thicket of Acacia seyal in Dinder National Park and the peri-domestic habitats of Umsalala and Ein-Elgamel villages, eastern Sudan; M, DNA molecular weight marker; lane 1, sample from mongoose captured in 1998 in DNP; lane 2, sample from mongoose captured in 1999 in peri-domestic habitat of Umsalala village; lane 3, sample from Mastomys natalensis captured in 1998 in DNP; lane 4, sample from Arvicanthis niloticus captured in 1999 in DNP; lane 5, DNA of $10^{2}$ promastigotes of $L$. donovani (MON18); lanes 6 and 7, PCR products from 2 samples of $\mathrm{H}_{2} \mathrm{O}$ (negative control).

shown in Fig. 2. A highly positive $808 \pm 12$ bp band, corresponding to L. donovani, was clear on the PCR product obtained from amplifying the DNA obtained from the spleen sample of the mongoose captured from Dinder in 1998. A similar $L$. donovani-specific band was noticeable in the products of the PCR done on the DNA obtained from the spleen of the second mongoose captured from the village peri-domestic habitat in 1999, and the 2 rodents ( $A$. niloticus and $M$. natelensis) captured from Dinder in 1998. However, the L. donovani specific band in samples from these 3 animals was extremely faint.

Prevalence of $\mathrm{L}$. donovani infection in $\mathrm{P}$. orientalis collected from DNP

High infection rates of Leishmania promastigotes in $P$. orientalis were observed during the two seasons. Of the $184 P$. orientalis females dissected in March 1998, there were 7 infected (3.4\% infection rate). In May 1999, a similar infection rate of $3.2 \%$ (5 out of 157 flies) of L. donovani in P. orientalis was detected. The PCR analysis confirmed that the flies were infected by L. donovani.

\section{DISCUSSION}

Lack of knowledge on the animal reservoir hosts of L. donovani in Sudan and other parts of East Africa is considered to be one of the biggest remaining gaps in our understanding of the epidemiology of visceral leishmaniasis (Ashford, 1996). This information will help not only in planning control measures of the disease in this region, but will also provide basic data on the molecular diversity of L. donovani. Furthermore, knowing the reservoir hosts of VL will help in establishing an appropriate experimental laboratory model, which can be used to study the biology of the parasite and understand the immunology of the disease.

In this paper, we report for the first time, the presence of $L$. donovani infection in the Egyptian Mongoose (Herpestes ichneumon). Due to the limitation encountered in animal trapping, it was not possible to examine larger numbers of the mongoose and hence estimate its rate of infection by $L$. donovani. However, the fact that we found 2 infected individuals in a total of 14 mongooses captured in 2 separate seasons and areas indicates that the infection may be quite common in this animal.

We suggest that the Egyptian mongoose is a possible reservoir host of $L$. donovani in eastern Sudan and probably other areas of East Africa. This animal appears to satisfy many criteria required for VL reservoir hosts (Ashford, 1996), including abundance, distribution, longevity, gregarious behaviour and possible close association with the vector. The Egyptian mongoose is widely distributed over the African Savannah (Kingdon, 1997) and appears to be quite abundant in the VL endemic zone of Sudan. The natural longevity of the mongoose is not known, but some individuals have been reported to survive up to 13 years in captivity. This animal, which is diurnal in activity, is known to exploit termite mounds, tree hollows (cavities) and soil cracks for siestas and night rest (Palmores \& Delibes, 1993). Although the resting behaviour of $P$. orientalis is not 
well understood, all possible sites recorded for the species seem to correspond to those used by the mongoose, e.g. tree cavities and old termite mounds (Elnaiem et al. 1997). It is probable that the vector comes in close prolonged contact with mongooses when the animal is resting in these habitats.

Another new host of L. donovani found was Mastomys natelensis, which was captured in DNP. The role of this species in the epidemiology of VL was previously intensively investigated by the NAMRU-3 team, who found no Leishmania infection in more than 1248 individuals of this species (Hoogstraal \& Heyneman, 1969). Due to the low number of $M$. natelensis sampled, we did not manage to estimate its infection rate with $L$. donovani. However, in light of the previous extensive experience of the NAMRU-3 group and the low abundance of the species in our study sites, it seems that $M$. natalensis is not an important reservoir for the infection.

Our finding of L. donovani infections in A. niloticus corresponds to the previous reports of Hoogstraal \& Heyneman (1969) and El Hassan et al. (1993). However, considering the small infection rate we found in this animal, we suggest that $A$. niloticus is an accidental host and is not a reservoir of the parasite. The significance of our findings on uninfected $A$. niloticus rats stems from the highly sensitive nested PCR techniques used, as well as the fact that we captured most of the animals from a site with a zoonotic cycle, which was proven by the finding of high infection rate in the vector (Elnaiem et al. 1998). The present results confirm these findings and show that the high infection rate in the vector remained stable over 4 years since the initial isolations which were made in 1995 and 1996. With such high and stable infection rates, we expect to find a correspondingly high association between the vector and the reservoir host, and consequently high infection rates of the parasite in the reservoir host. Similarly high infection rates $(7 \cdot 3 \%)$ of Leishmania in phlebotomine sandflies have been observed in Leishmania guyanensis/Lutzomyia umbratilis in Brazil, where there is a close association on tree trunks and canopies between the vector and reservoir sloths (Lainson, Ward \& Shaw, 1976; Lainson, 1983).

There is little information available about the natural hosts of $P$. orientalis. However, in a previous host preference study, light traps baited with $A$. niloticus did not seem to have improved the collection as compared to light traps without the animal (Elnaiem, D. A., Hassan, K. H. \& Killick-Kendrick, R., unpublished results). Similarly, in previous attempts to establish colonies of $P$. orientalis, we had repeated failures in stimulating wild-caught females to feed on $A$. niloticus captured from the area, indicating that $A$. niloticus is not a natural host of $P$. orientalis.
Further work is needed to determine the actual infection rate of $L$. donovani in the mongoose and other possible VL reservoir hosts and to correlate the zymodemes of the parasite found in animals with those present in VL patients and sandflies. Future investigations are also needed to study the host preference of the vector and its possible association with the mongoose and other animals.

Our warm thanks are due to Miss I. Mauricio and Dr I. Frame (London School of Hygiene) for their great help and guidance. We are grateful to Miss D. Hills and Dr P. Jenkins (British Natural History Museum, London) for their help in identification of small mammals. We thank Dr H. Noyes (University of Liverpool), and Dr B. Lambson, Miss S. McCann and Professor D. Barker (Cambridge University) for their help with training on PCR techniques. We are grateful to Professor A. M. El Hassan, Dr D. Musa and Dr O. Osman (University of Khartoum), M. Ponce and D. Nimmo (Keele University) and staff of the Wild Life Conservation Department (Sudan) for their help and support. This study received financial support from the Wellcome Trust (Research Travelling Training Fellowship No. 052211/Z/97/Z/MEP/JPS/CRD for Dr D. A. Elnaiem) and the UNDP/World Bank/WHO Special Programme for Research and Training in Tropical Diseases (student small training grant number 990610 for Mr M. Hassan).

\section{REFERENCES}

ASHFORD, R. W. (1996). Leishmaniasis reservoirs and their significance in control. Clinical Dermatology 14, 523-532.

ASHFORD, R. W. \& BETTINI, s. (1987). Ecology and Epidemiology: Old World. In The Leishmaniasis in Biology and Medicine, Vol. 1 Biology and Epidemiology (ed. Peters, W. \& Killick-Kendrick, R.), pp. 366-424. Orlando Academic Press, Orlando, USA.

ASHFORD, R. W., SEAMAN, J., SCHORCHER, J. \& PRATLONG, F. (1992). Epidemic visceral leishmaniasis in southern Sudan: identity and systematic position of the parasites from patients and vectors. Transactions of the Royal Society of Tropical Medicine and Hygiene 86, 379-380.

COURTNey, o., Santana, E. W., Johnson, P. J., VASCONCELOS, I. A. B. \& VASCONCELOS, A. W. (1996). Visceral leishmaniasis in the hoary zorro Dusicyon vetulus: A case of mistaken identity. Transactions of the Royal Society of Tropical Medicine and Hygiene 90, 498-502.

DeUrere, J., Boni, M., PRATlong, F., OSMAN, M., BOUChetON, B., EL-SAFi, S., FEUGier, E., MUSA, M. K., DARoust, B., DESSEIN, A. \& DEDET, J. P. (2000). Visceral leishmaniasis in Sudan: First identification of Leishmania from dogs. Transactions of the Royal Society of Tropical Medicine and Hygiene 94, 154-155. Desjeux, P. (1996). Leishmaniasis. Public health aspects and control. Clinical Dermatology 14, 417-423.

EL Hassan, A. M., ZijLSTRA, E. E., MEREDith, s. E. O. \& ISMAIL, A. (1993). Identification of Leishmania donovani, using a polymerase chain reaction in patients and animal material from an area of endemic kala-azar in the Sudan. Acta Tropica 55, 87-90. 
ELNAIEM, D. A., HASSAN, H. K. \& WARD, R. D. (1997). Phlebotomine sandflies in a focus of visceral leishmaniasis in a border area of eastern Sudan. Annals of Tropical Medicine and Parasitology 91, 307-318.

ELNAIEM, D. A. \& OSMAN, O. F. (1998). Evidence for active transmission of visceral leishmaniasis within a village in eastern Sudan. Acta Tropica 71, 305-309.

ELNAIEM, D. A., WARD, R. D., MILES, M. A. \& FRAME, I. A. (1998). Infection rates of Leishmania donovani in Phlebotomus orientalis from a visceral leishmaniasis focus in eastern Sudan. Annals of Tropical Medicine and Parasitology 92, 229-232.

HOOGSTRAal, H. \& HEYNEMANN, D. (1969). Leishmaniasis in the Sudan Republic. 30. Final epidemiological report. American Journal of Tropical Medicine and Hygiene 18, 1091-1210.

KILLICK-KENDRICK, R. (1992). Phlebotomine vectors of the leishmaniasis. A review. Medical and Veterinary Entomology 41, 1-24.

kingdon, J. (1997). The Kingdon Field Guide to African Mammals. Academic Press, Harcourt Brace and Company, London.

KIRK, R. (1939). Studies in leishmaniasis in the AngloEgyptian Sudan. Part 1. Epidemiology and general considerations. Transactions of the Royal Society of Tropical Medicine and Hygiene 42, 533-544.

LAInson, R. (1983). The American leishmaniasis: Some observations on their ecology and epidemiology. Transactions of the Royal Society of Tropical Medicine and Hygiene 77, 569-596.

LAINSON, R., SHAW, J. J. \& LINS, Z. C. (1969). Leishmaniasis in Brazil: IV. The fox, Cerdocyon thous (L.) as a reservoir of Leishmania donovani in Pará State, Brazil. Transactions of the Royal Society of Tropical Medicine and Hygiene 63, 741-745.

LAINSON, R., WARD, R. \& SHAW, J. J. (1976). Cutaneous leishmaniasis in north Brazil: Lutzomyia anduzei as a major vector. Transactions of the Royal Society of Tropical Medicine and Hygiene 70, 171-172.

NOYES, H. A., REYBURN, H., BAILEY, J. W. \& SMith, D. (1998). A nested-PCR-based schizodeme method for identifying Leishmania kinetoplast minicircle classes directly from clinical samples and its application to the study of the epidemiology of Leishmania tropica in Pakistan. Fournal of Clinical Microbiology 36, 2877-2881.
OSKaM, L., Pratlong, F., Zijlstra, E. E., KROON, C. C. M., DEDET, J.P., KAGER, P. A., SCHONIAN, G., GHALIB, H. W., EL HASSAN, A. M. \& MEREDith, S. E. O. (1998).

Biochemical and molecular characterization of Leishmania parasites isolated from an endemic focus in eastern Sudan. Transactions of the Royal Society of Tropical Medicine and Hygiene 92, 120-122.

PALMORES, F. \& DELIBES, M. (1993). Resting ecology and behaviour of Egyptian mongooses (Herpestes ichneumon) in southwestern Spain. Fournal of Zoology 230, 557-566.

PETERS, W. \& KILLICK-KENDRICK, R. (1987). The Leishmaniasis in Biology and Medicine, Vols I and II. Orlando Academic Press, Orlando, USA.

SCHORSCHER, J. A. \& GORIS, M. (1992). Incrimination of Phlebotomus (Larroussius) orientalis as a vector of visceral leishmaniasis in Western Upper Nile Province, southern Sudan. Transactions of the Royal Society of Tropical Medicine and Hygiene 86, 622-623. SEAMANn, J., MERCER, A. J. \& SONdorp, E. (1996). The epidemic of visceral leishmaniasis in Western Upper Nile, Southern Sudan: course and impact from 1984 to 1994. International Fournal of Epidemiology 25 , 862-971.

SIXL, W., SIXL, B., ABDEL-NABI, O. \& SEBEK, Z. (1987). Contribution to the problem of the diagnosis of leishmaniasis in south Sudan. Geographical Medicine 5, 189-203.

SMYTH, A. J., GHOSH, A., HASSAN, M. Q., BASU, D., DE BRUiJN, M. H. L., ADHAYA, S., MALIK, K. K. \& BARKER, D. (1992). Rapid and sensitive detection of Leishmania kinetoplast DNA from spleen and blood samples of kala-azar patients. Parasitology 105, 183-192.

THOMSON, M. C., ELNAIEM, D. A., ASHFORD, R. W. \& CONNOR, s. J. (1999). The development of a kala-azar risk map for Sudan: mapping the potential distribution of Phlebotomus orientalis using digital data of environmental variables. Tropical Medicine and International Health 4, 105-113.

Zijlstra, E. E., El Hassan, A. M., GHAlib, H. W. \& ISMAEL, A. (1994). Endemic kala-azar in eastern Sudan: a longitudinal study on the incidence of clinical and sub-clinical disease and post-kala-azar dermal leishmaniasis. American Fournal of Tropical Medicine and Hygiene 51, 826-836. 